\title{
Magnetization plateaus in frustrated antiferromagnetic quantum spin models
}

\author{
A. Honecker $\dagger \|$, J. Schulenburg $\ddagger$ and J. Richter $\uparrow$ \\ $\dagger$ Institut für Theoretische Physik, Technische Universität Braunschweig, 38106 \\ Braunschweig, Germany \\ $\ddagger$ Universitätsrechenzentrum, Otto-von-Guericke-Universität Magdeburg, 39016 \\ Magdeburg, Germany \\ I Institut für Theoretische Physik, Otto-von-Guericke-Universität Magdeburg, 39016 \\ Magdeburg, Germany
}

\begin{abstract}
Plateaus can be observed in the zero-temperature magnetization curve of quantum spin systems at rational values of the magnetization. In one dimension, the appearance of a plateau is controlled by a quantization condition for the magnetization which involves the length of the local spin and the volume of a translational unit cell of the ground state. We discuss examples of geometrically frustrated quantum spin systems with large (in general unbounded) periodicities of spontaneous breaking of translational symmetry in the ground state.

In two dimensions, we discuss the square, triangular and Kagomé lattices using exact diagonalization (ED) for up to $N=40$ sites. For the spin- $1 / 2 X X Z$ model on the triangular lattice we study the nature and stability region of a plateau at one third of the saturation magnetization. The Kagomé lattice gives rise to particularly rich behaviour with several plateaus in the magnetization curve and a jump due to local magnon excitations just below saturation.
\end{abstract}

Submitted to: J. Phys.: Condens. Matter

13 September 2003

PACS numbers: 75.10.Jm, 75.45.+j, 75.50.Ee, 75.60.Ej

\section{Introduction}

Geometrically frustrated quantum spin systems constitute a class of systems exhibiting interesting quantum phenomena like different (unusual) quantum phases (see e.g. [1, 2] for reviews of two-dimensional models). An external magnetic field may further enhance this frustration since it competes with the antiparallel alignment of spins favoured by antiferromagnetic exchange. This leads to interesting quantum phenomena in the high-field magnetization process of geometrically frustrated quantum spin systems. In particular, a spin gap may be opened by the external magnetic field, giving rise to a plateau in the $T=0$ magnetization curve. Although no complete review of the subject

\| To whom correspondence should be addressed (a.honecker@tu-bs.de) 
exists so far, different aspects are summarized e.g. in [1, 2, 3, 4, 5]. In this article we will illustrate the status of the field by pointing out and presenting selected recent and new results.

Specifically we consider the $X X Z$ model in an external magnetic field $h$

$$
H=\sum_{\langle i, j\rangle} J_{i, j}\left(S_{i}^{x} S_{j}^{x}+S_{i}^{y} S_{j}^{y}+\Delta S_{i}^{z} S_{j}^{z}\right)-h \sum_{i} S_{i}^{z}
$$

where the $\vec{S}_{i}$ are spin-S operators at site $i$ and the $J_{i, j}$ are the exchange constants between pairs of sites $\langle i, j\rangle$ connected in the lattice topology under consideration. The special case of (11) where the $X X Z$ anisotropy satisfies $\Delta=1$ corresponds to the Heisenberg model. Below we will concentrate on the extreme quantum limit $S=1 / 2$ and antiferromagnetic coupling $J_{i, j} \geq 0$ although some of the results can be generalized e.g. to $S>1 / 2$.

An important observable is the magnetization

$$
\langle M\rangle=\frac{1}{N S}\left\langle\sum_{i} S_{i}^{z}\right\rangle
$$

which we normalize to saturation value $\langle M\rangle=1$ (here $N$ is the total number of spins in the system). Note that the magnetization (2) is a conserved quantity for the Hamiltonian (11). This is technically useful for computing the magnetization curve e.g. by exact diagonalization (ED) using the Lanczos method.

\section{One dimension}

In one dimension, Oshikawa, Yamanaka and Affleck proposed the following condition on the magnetization $\langle M\rangle$ at a plateau in the $T=0$ magnetization curve [6]

$$
S V(1-\langle M\rangle) \in \mathbb{Z}
$$

Here $S$ is the size of the local spin and $V$ is the number of spins in a translational unit cell of the ground state. The condition (3) can be understood easily starting from a limit where the system decouples into clusters with $V$ sites (see e.g. [7) and in this case $V$ is the number of spins in a unit cell of the Hamiltonian. However, in particular in frustrated spin systems one finds that translational symmetry is spontaneously broken in the ground state (c.f. examples in the following sections) and then $V$ is larger than (namely an integer multiple of) the unit cell of the Hamiltonian. Nevertheless, the quantization condition (3) implies that the magnetization $\langle M\rangle$ always has a rational value on a plateau.

\subsection{Frustrated $S=1 / 2$ Heisenberg chain}

Let us start by discussing some selected aspects of the $S=1 / 2$ frustrated Heisenberg chain (see inset of Fig. 11). Majumdar and Ghosh noticed more than 30 years ago [8, 9, 10, that one can explicitly write down the ground state if the next-nearest neighbour 


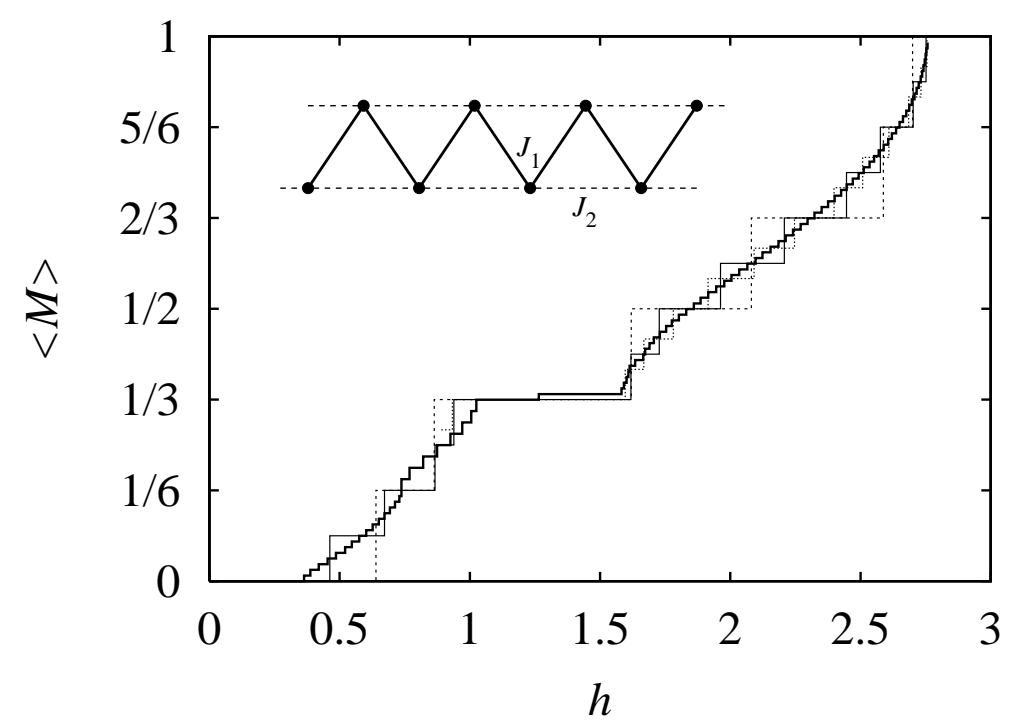

Figure 1. Inset: Heisenberg chain with nearest and next-nearest neighbor coupling $J_{1}$ and $J_{2}$, respectively. Points denote the locations of $S=1 / 2$ spins. Main panel: Magnetization curve for $J_{1}=1, J_{2}=0.8$. The thin dashed and solid curves were obtained by ED of rings (periodic boundary conditions) with $L=12$ (dashed), 24 (full) [12] and 36 sites (dotted); the bold solid curve was obtained by DMRG for $L=192$ sites with open boundary conditions [13].

coupling $J_{2}$ and the nearest neighbour coupling $J_{1}$ are related by $J_{2}=J_{1} / 2$. This so-called 'Majumdar-Ghosh' state is a two-fold degenerate dimer product state and has become a paradigm for the frustration-induced opening of a spin gap accompanied by spontaneous breaking of translational symmetry by a period two.

In the presence of a magnetic field, the spin gap leads to an $\langle M\rangle=0$ plateau in the magnetization curve. Starting with [1], many authors have studied the magnetization process of this model by ED (see e.g. [12] and references therein) and no plateaus with $\langle M\rangle \neq 0$ were found. Finally, a recent density-matrix renormalization group (DMRG) study of $S=1 / 2$ frustrated Heisenberg chain exhibited a clear plateau at $\langle M\rangle=1 / 3$ in the region of strong frustration $J_{1} \approx J_{2}[13$, as is illustrated by the main panel of Fig. 11 which reproduces the magnetization curve for $J_{2}=0.8 J_{1}$ (the step on the $\langle M\rangle=1 / 3$ plateau in the DMRG curve is due to the open boundaries). The state of this $\langle M\rangle=1 / 3$ plateau spontaneously breaks translational symmetry by a period three. Two points are worthwhile noticing here. First, this period three is higher than the period two appearing in the Majumdar-Ghosh state [8, 9, 10] at $h=0$. Second, the very presence of this plateau was missed for a long time.

With hindsight, this $\langle M\rangle=1 / 3$ plateau can be observed also in the ED results for $L=12$ and 24 sites and periodic boundary conditions (reproduced for $J_{2}=0.8 J_{1}$ in Fig. 1 after [12 - this figure also includes a new $L=36$ curve which extends below the $\langle M\rangle=1 / 3$ plateau). It may be instructive to consider the reasons for missing it nevertheless. First, the excited states at $h=0$ [14] and correspondingly the ground 


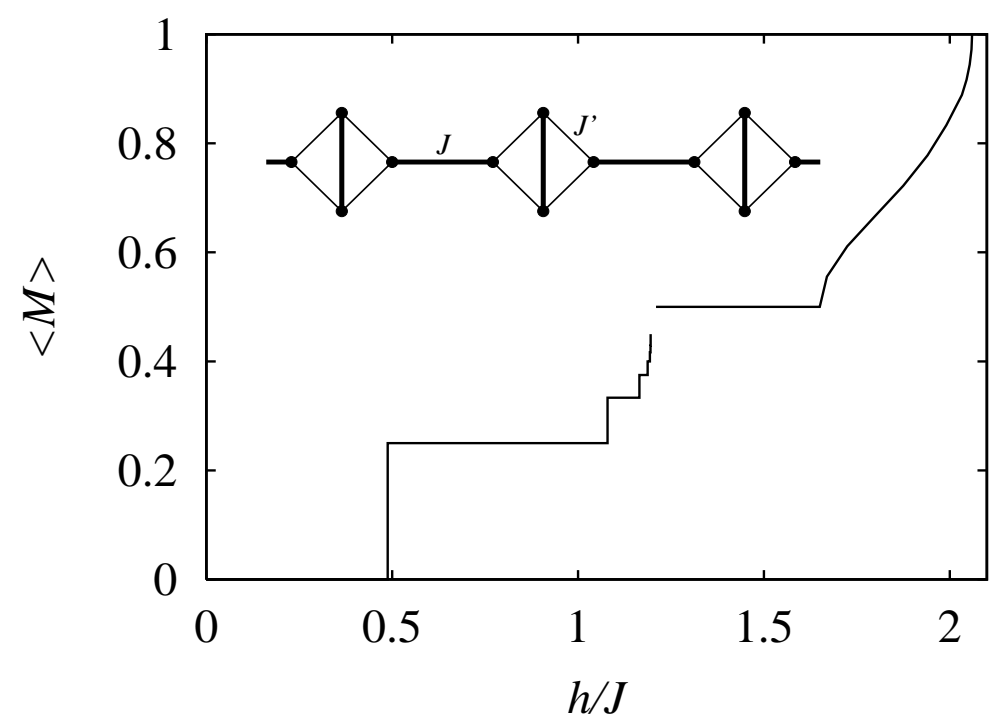

Figure 2. Inset: Orthogonal dimer chain. Points denote the locations of $S=1 / 2$ spins. Main panel: Magnetization curve for $J^{\prime}=0.7 J$ 19. Note the long (probably infinite) sequence of plateaus for $\langle M\rangle<1 / 2$.

states in a magnetic field $h \neq 0$ have in general incommensurate momenta [12] which leads to oscillating finite-size effects. Second, one should concentrate on system sizes that can accommodate the known antiferromagnetic ground states in the limit of two decoupled chains $J_{1} \rightarrow 0$, implying that $L$ should be a multiple of 4 . In addition, only those values of the magnetization can be studied which are realized for a given system size. In particular, $\langle M\rangle=1 / 3$ is realized only if $L$ is a multiple of 3 . These considerations restrict the system sizes that should be used for the discussion of an $\langle M\rangle=1 / 3$ plateau in the frustrated Heisenberg chain to multiples of 12 . The first cases are $L=12,24$ and 36. These are used in Fig. 1 and are in good agreement with the DMRG results of [13].

\subsection{Orthogonal dimer chain}

Now we wish to draw the reader's attention to the $S=1 / 2$ orthogonal dimer chain (also known as dimer-plaquette chain) [15, 16] shown in the inset of Fig. 2] a further one-dimensional model where some remarkable properties were missed originally. This model attracted renewed attention recently since it can be regarded as a one-dimensional version of the two-dimensional Shastry-Sutherland model [17].

A first study [18 of the magnetization process of the $S=1 / 2$ orthogonal dimer chain found plateaus with $\langle M\rangle=1 / 4$ and $1 / 2$. However, this study missed further plateaus and thus the remarkable property of the orthogonal dimer chain that it gives rise to a magnetization curve with (probably infinitely) many plateaus for some values of the parameters [19]. At least some steps in the derivation can be carried out exactly exploiting the special property of the orthogonal dimer chain that the total spin on 
each vertical dimer is conserved. A plateau-state can then be characterized as follows 19]: $k$ consecutive vertical dimers form triplets $(S=1)$, separated by singlets $(S=0)$. Covering the chain periodically with such 'fragments' of length $k$ each in the sector $S^{z}=k$ yields

$$
\langle M\rangle=\frac{k}{2 k+2} .
$$

At $J^{\prime}=0.7 J>0$ and for $\langle M\rangle<1 / 2$ only precisely these states consisting of fragments of length $k$ with $S^{z}=k$ appear as ground states in a magnetic field [19], whereas for $\langle M\rangle>1 / 2$ the ground state is in a sector where all vertical dimers form triplets $S=1$ (one difference between the present Fig. 2] and the original Fig. 3 of [19] is in the system sizes that have been used for extrapolating the magnetization curve in the region $\langle M\rangle \geq 1 / 2$ ). As long as $\langle M\rangle<1 / 2$, fragments of increasing length become ground states with increasing field, leading to a magnetization curve with infinitely many plateaus at the magnetization values given by (4) and jumps in between, compare the main panel of Fig. 2, This infinite series accumulates at $\langle M\rangle=1 / 2$, where one finds a further pronounced plateau before a smooth transition to saturation follows.

The infinite sequence of plateaus arises in the orthogonal dimer chain because the ground states manifestly break translational symmetry with arbitrarily long periodicity $k+1$, implying that at least in one dimension there is no general upper bound on the possible periods for spontaneous breaking of translational symmetry.

\section{Two dimensions}

To the best of our knowledge there are no rigorous arguments why the condition (3) should hold in dimensions higher than one. Furthermore, starting from two dimensions one may have true long-range order in the spin components at $T=0$. Accordingly, an external magnetic field can induce transitions between different ordered and disordered states.

Already in one dimension, a plateau at a given $\langle M\rangle$ still requires a detailed analysis of the specific model. Even more so, the presence and nature of a plateau state must be discussed case-by-case in two dimensions. Some selected examples are presented in the following sections.

\subsection{Square lattice}

Let us start with a brief discussion of the magnetization process of the square lattice which is a non-frustrated model and well understood. Fig. 3 shows the magnetization curve of the $S=1 / 2$ square-lattice Heisenberg model $(\Delta=1)$ obtained by different approaches. First, the thin full line shows the result obtained by ED for a finite lattice with $N=40$ sites (see also [20, 21] for earlier ED studies - in the particular case $N=40$, the largest dimension to be treated was 554596326 and occurred in the sector $S^{z}=3$ ). The full bold line denotes an extrapolation of the ED data to the thermodynamic limit 


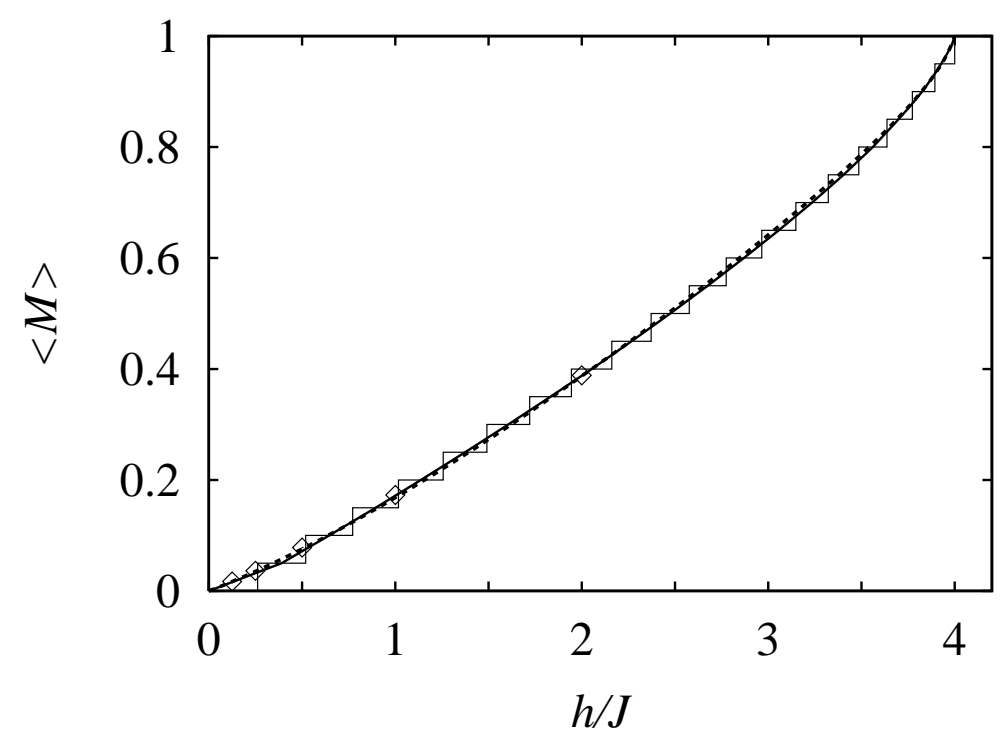

Figure 3. Magnetization curve of the $S=1 / 2$ Heisenberg antiferromagnet on the square lattice. The thin solid line is for $N=40$ sites, the full bold line is an extrapolation to the thermodynamic limit. Also shown are a second-order spinwave result 22] (dashed bold line) and QMC results 223] (diamonds).

which is obtained by connecting the midpoints of the finite-size steps at the largest available system size. One observes a smooth magnetization curve with no peculiar features (in particular no plateaus) for $|\langle M\rangle|<1$. Note that close to saturation the extrapolated curve includes data at large system sizes, which is not shown explicitly in Fig. 3. More precisely, for $\langle M\rangle \geq 0.84375$, the curve is based exclusively on finite lattices with at least $8 \times 8$ sites. The high-field part of the magnetization curve is therefore particularly well controlled by ED.

Second, a second-order spinwave result [22] is also available and shown by the bold dashed line in Fig. 3. Third, the magnetization process of the square lattice can also be studied by quantum Monte Carlo (QMC) since this lattice is not frustrated. The diamonds in Fig. [3 show available stochastic-series-expansion QMC results [23].

The quantitive differences of the results of all three approaches are small, i.e. each approach yields a good description of the $S=1 / 2$ square lattice. Since the spinwave approach 22] is based on a Néel state, we may therefore conclude that Néel order prevails in the transverse components for $|\langle M\rangle|<1$ (see also [20] for a discussion from the point of view of $\mathrm{ED})$.

\subsection{Triangular lattice}

The $S=1 / 2 X X Z$ model on the triangular lattice is among the first models whose magnetization process was studied by ED [24]. These early studies already found a plateau with $\langle M\rangle=1 / 3$, at least for Ising-like anisotropies $\Delta>1$. Due to the restriction to at most 21 sites, it was first not completely clear whether the plateau persists in the 


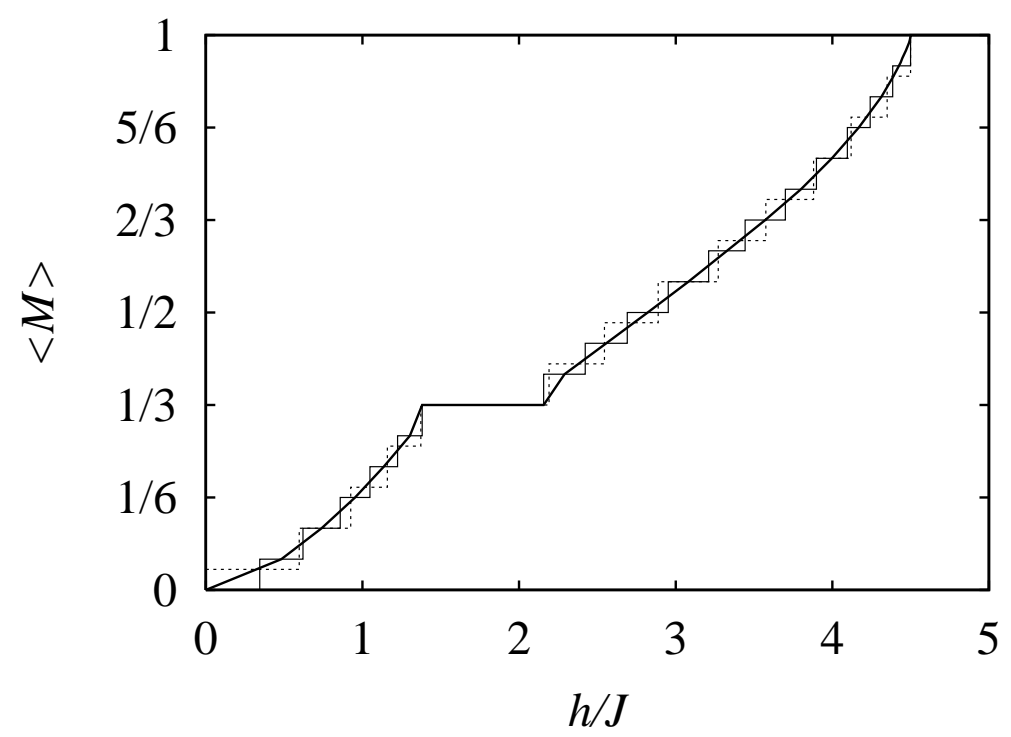

Figure 4. Magnetization curve of the $S=1 / 2$ Heisenberg antiferromagnet on the triangular lattice. The thin dashed and solid line are for $N=27$ and $N=36$ sites, respectively. The bold line is an extrapolation to the thermodynamic limit.

isotropic regime $\Delta \approx 1$. The magnetization process of the isotropic model was analyzed further using spinwave theory [25]. This study provided evidence that the $\langle M\rangle=1 / 3$ plateau exists also at $\Delta=1$ and estimates for its boundaries were obtained.

Fig. 4 shows the magnetization curves obtained by ED for $\Delta=1$ on finite lattices with $N=27$ and 36 sites (thin lines). Both curves exhibit a clear plateau at $\langle M\rangle=1 / 3$ in an otherwise smooth magnetization curve. These two curves overlap with previous ED results [21, 26]. Note, however, that for $N=36$ only the sector $\vec{k}=0$ was studied previously for $\langle M\rangle \leq 1 / 3$ [26], but it turns out that the ground state is not always in this sector. Inclusion of sectors with $\vec{k} \neq 0$ for $N=36$, a different shape of the $N=27$ system and a previously incomplete $N=36$ curve lead to small differences of our Fig. [4 as compared to Fig. 4 of [21]. The first-order spinwave results for the magnetic fields at the lower and the upper boundaries of the $\langle M\rangle=1 / 3$ plateau [25] are smaller by about $0.13 J$ (lower boundary) and $0.01 J$ (upper boundary) than the ED results presented here for $N=36$ and $S=1 / 2$.

The full bold line in Fig. 团 denotes an extrapolation of the ED data to the thermodynamic limit which is obtained by connecting the midpoints of the finite-size steps at the largest available system size (except for the boundaries of the $\langle M\rangle=1 / 3$ plateau where corners were used). Close to saturation this includes again bigger system sizes than those explicitly shown in Fig. 4.

The state of the $\langle M\rangle=1 / 3$ plateau can be easily understood in the Ising limit $\Delta \gg 1$ [27, 21]. Quantum fluctuations are completely suppressed in the limit $\Delta \rightarrow \infty$ and the $\langle M\rangle=1 / 3$ state is a classical state where all spins on two of the three sublattices of the triangular lattice point up and all spins on the third sublattice point down, as is 


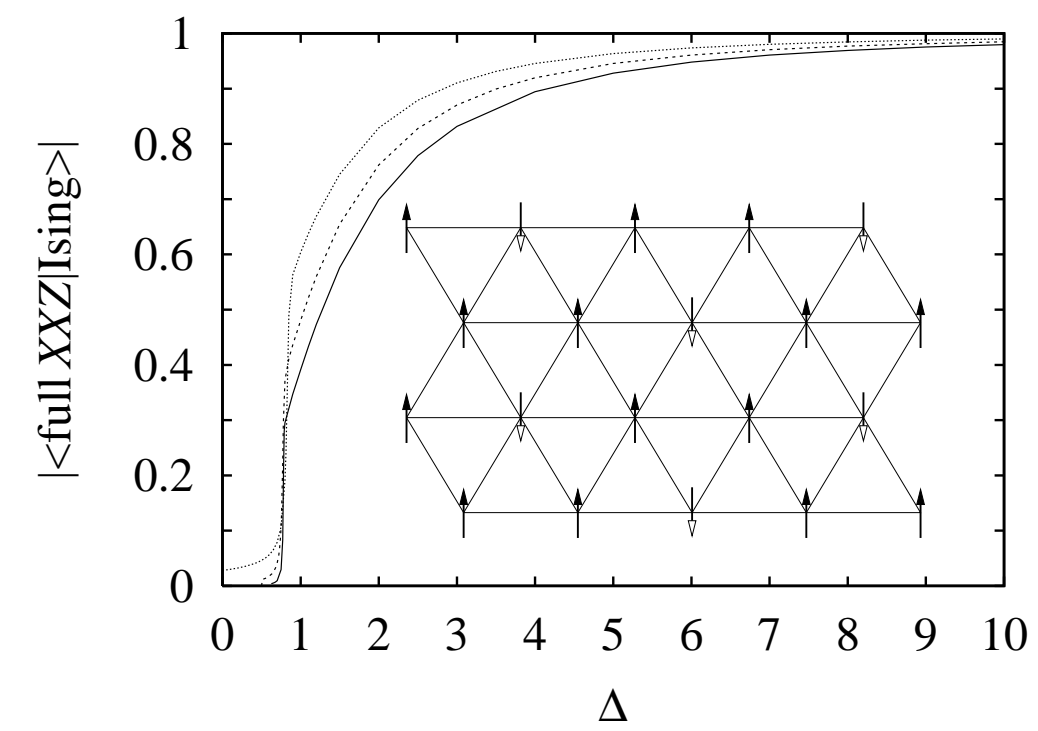

Figure 5. Overlap of the wave function $\mid$ full $X X Z\rangle$ of the full $S=1 / 2 X X Z$ model on the triangular lattice at $\langle M\rangle=1 / 3$ with the corresponding state $\mid$ Ising $\rangle$ of the Ising model as a function of the $X X Z$ anisotropy $\Delta$. Lines are for finite lattices with $N=18$ (dotted), 27 (dashed) and 36 (full) sites. The inset shows one representative state $\mid$ Ising $\rangle$ for the three degenerate ground states of the Ising model at $\langle M\rangle=1 / 3$.

sketched in the inset of Fig. 5. This state corresponds to an ordered collinear spin configuration. It breaks translational symmetry by a period three and accordingly is threefold degenerate. One can then use perturbation theory in $1 / \Delta$ to study the $\langle M\rangle=1 / 3$ plateau of the $X X Z$ model [21]. Here we numerically compute the overlap of the appropriate linear combination |Ising $\rangle$ of the three Ising states and the $\langle M\rangle=1 / 3$ wave function $\mid$ full $X X Z\rangle$ of the full $X X Z$ model with $S=1 / 2$ in order to show that this description remains qualitatively valid even in the isotropic region $\Delta \approx 1$. Fig. 5 shows results for the overlap $\mid\langle$ full $X X Z|$ Ising $\rangle \mid$ on finite lattices with $N=18,27$ and 36 sites (for $N=36$ we have computed the overlaps by considering only the wave function within the $\vec{k}$-subspace that contains the ground state for $\Delta \geq 1$ ). One observes that this overlap remains large even in the vicinity of the Heisenberg model $\Delta=1$ and drops sharply around $\Delta=0.75$. Accordingly, this $\langle M\rangle=1 / 3$ plateau state is a stable phase for $\Delta>\Delta_{c}$ with $\Delta_{c} \approx 0.75$. Remarkably, this estimate for $\Delta_{c}$ is close to the estimate $\Delta_{c} \approx 0.85$ for the vanishing point of the $\langle M\rangle=1 / 3$ plateau which was obtained in [21] by comparing the plateau widths on $3 \times 6$ and $3 \times 9$ systems. Note that the location of the sharp drop in Fig. $[5$ is almost independent of system size although the absolute values of the overlaps $\mid\langle$ full $X X Z|$ Ising $\rangle \mid$ do depend on $N$. We can thus locate the point $\Delta_{c}$ where the $\langle M\rangle=1 / 3$ plateau disappears quite accurately at $\Delta_{c}=0.76 \pm 0.03$. 


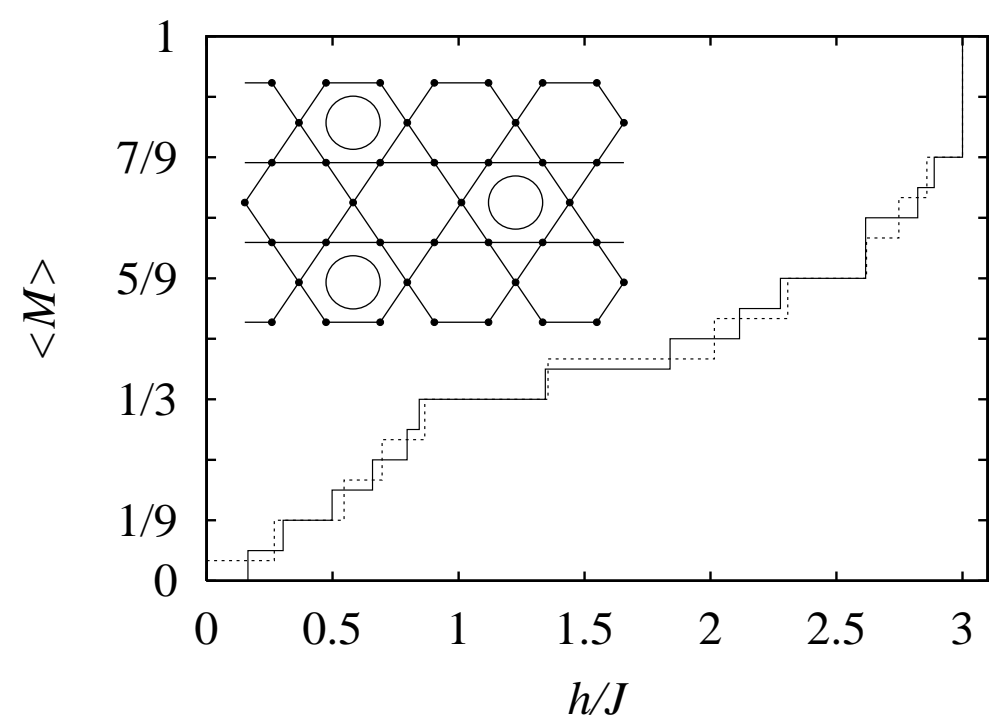

Figure 6. Magnetization curve of the $S=1 / 2$ Heisenberg antiferromagnet on the Kagomé lattice with $N=27$ (dashed line) and 36 sites (solid line). The inset shows part of the Kagomé lattice with a $\sqrt{3} \times \sqrt{3}$ superstructure indicated by the circles in certain hexagons.

\subsection{Kagomé lattice}

Finally, Fig. [6 shows magnetization curves for the Kagomé lattice (see inset) with $N=27$ [28, 29] and 36 sites. Note that the $N=36$ curve in Fig. [6] differs slightly from the corresponding curve in [29] for $\langle M\rangle \leq 1 / 3$. The reason is that due to the computational effort only selected $\vec{k}$-sectors were investigated in [29] and in some cases the correct symmetry of the ground state was in fact missed. Our new $N=36$ curve agrees with unpublished results of Waldtmann and Everts [30].

The Kagomé lattice is famous for a disordered ground state at $h=0$ accompanied by a small spin gap with many singlets inside this gap (see 31, 32 and references therein). The spin gap should give rise to a narrow $\langle M\rangle=0$ plateau even if it is difficult to recognize in Fig. 6.

A plateau at $\langle M\rangle=1 / 3$ can be recognized better in Fig. [6] In fact, the presence of this plateau at $\langle M\rangle=1 / 3$ in the $S=1 / 2$ Heisenberg model on the Kagomé lattice has been established previously by considering also system sizes different from those shown in Fig. [6 [28, 33]. Nevertheless, the state of this plateau is still an interesting issue. For the classical Heisenberg model at $\langle M\rangle=1 / 3$, thermal fluctuations select collinear states, but due to the huge degeneracy of this space, there appears to be no real order on the classical level at $\langle M\rangle=1 / 3$ [34] (see also [35]). For $S=1 / 2$, it is useful to consider the $X X Z$ model. In the Ising limit $\Delta \rightarrow \infty$ one can then first establish [36] a relation to a quantum dimer model on the hexagonal lattice which was argued [37, 38] to give rise to a valence bond crystal ground state with a $\sqrt{3} \times \sqrt{3}$ order indicated by the circles in the hexagons in the inset of Fig. 6. Furthermore, like for the triangular lattice one can 
compute the overlap (see Fig. 5) of the $\langle M\rangle=1 / 3$ wave function of the $X X Z$ model with that of the quantum dimer model as a function of $\Delta$ and one finds no evidence for a phase transition for $\Delta \geq 1$ [36]. This implies that also the $\langle M\rangle=1 / 3$ state of the $S=1 / 2$ Heisenberg model on the Kagomé lattice is a three-fold degenerate valence bond crystal, i.e. an ordered state with many low-lying non-magnetic excitations, but still a small gap to all excitations [36].

There may be a further plateau at $\langle M\rangle=5 / 9$ in Fig. [6] although it is difficult to draw unambiguous conclusions from the available numerical data in this region of magnetization values.

Finally, one can see a pronounced jump of height $\delta\langle M\rangle=2 / 9$ just below saturation in Fig. 6. This jump arises due to local magnon excitations which can be constructed in the spin- $S X X Z$ model on the Kagomé lattice [29, 39]. These local magnon excitations give rise to an exact degeneracy at the saturation field and thus a macroscopic jump in the magnetization curve of height $\delta\langle M\rangle=1 /(9 S)$. In the state just below the jump, magnons are localized on the hexagons marked by circles in the inset of Fig. 6. Since this is a crystalline ordered state, general arguments (see e.g. [40, 41]) predict a gap and consequently a plateau in the magnetization curve at $\langle M\rangle=1-1 /(9 S)$. Indeed, a plateau at $\langle M\rangle=7 / 9$ can be seen clearly in the magnetization curve of the $S=1 / 2$ Heisenberg model on the Kagomé lattice in particular when one considers also lattices with $N=45$ and 54 sites [29].

\section{Conclusions and outlook}

In this article we have discussed examples of plateaus in one- and two-dimensional quantum spin models. In one dimension, the appearance of a plateau is controlled by the quantization condition (3) which implies in particular that the magnetization $\langle M\rangle$ on a plateau must be rational. This condition can be interpreted as a commensurability condition for the ground state. Hence, it is important to observe that translational symmetry can be spontaneously broken in frustrated quantum spin models. Even in well-studied examples such as the frustrated $S=1 / 2$ Heisenberg chain, an $\langle M\rangle=1 / 3$ plateau with a spontaneously broken period three was discovered only recently [13]. Furthermore, an infinite sequence of plateaus in the orthogonal dimer chain [19] shows that there is no upper bound on the possible periodicity of spontaneous breaking of translational symmetry.

It is less clear if the condition (3) is also applicable to two and higher dimensions. Nevertheless, it works for most examples discussed here: The state of the $\langle M\rangle=1 / 3$ plateau in the $S=1 / 2$ triangular lattice has a unit cell with $V=3$ spins (see section 3.2) and for the Kagomé lattice the plateaus with $\langle M\rangle=1 / 3$ in the $S=1 / 2$ model as well as the one with $\langle M\rangle=1-1 /(9 S)$ in the spin- $S$ model both have unit cells with $V=9$ spins (see section [3.3, in particular the inset of Fig. 6). These cases fit well with the quantization condition (3). However, the disordered ground state at $h=0$ in the $S=1 / 2$ Kagomé lattice (see [31, 32] and references therein) is not covered by the 
condition (3) since it gives rise to an $\langle M\rangle=0$ plateau without the appropriate breaking of translational symmetry.

From the point of view of experiments, the exchange constants $J_{i, j}$ dictate the scale of magnetic fields needed to access the high-field region. $\mathrm{SrCu}_{2}\left(\mathrm{BO}_{3}\right)_{2}$ is an $S=1 / 2$ system with sufficiently small $J$ 's that permit the observation of plateaus at $\langle M\rangle=1 / 8$, $1 / 4$ and $1 / 3$ in pulsed-field magnetization experiments [42, 43]. $\mathrm{SrCu}_{2}\left(\mathrm{BO}_{3}\right)_{2}$ is believed to be a good realization of the two-dimensional Shastry-Sutherland lattice [17]. Because of its relation to $\mathrm{SrCu}_{2}\left(\mathrm{BO}_{3}\right)_{2}$, this frustrated spin model has been analyzed theoretically in detail (see 44. for a recent review). We hope that the examples discussed in this article will stimulate further theoretical and experimental research on the magnetization process of frustrated quantum spin models.

\section{Acknowledgments}

We would like to thank D.C. Cabra, M.D. Grynberg, P.C.W. Holdsworth and P. Pujol for useful discussions. The more complicated computations presented in this article have been performed on the compute-servers cfgauss and wildfire at the computing centers of the TU Braunschweig and Magdeburg University, respectively. We are particularly grateful to J. Schüle of the Rechenzentrum at the TU Braunschweig for technical support. This work was partly supported by the DFG (project Ri615/10-1).

\section{References}

[1] Lhuillier C and Misguich G 2001 High Magnetic Fields Lecture notes in physics 595 eds. Berthier C, Lévy L P and Martinez G (Berlin: Springer) p 161 cond-mat/0109146; http://www.springerlink.com/link.asp?id=6xwapvm1g9x9by93

[2] Richter J, Schulenburg J and Honecker A 2003 in preparation

[3] Cabra D C, Grynberg M D, Honecker A and Pujol P 2001 Condensed Matter Theories vol 16 eds. Hernández S and Clark J W (New York: Nova Science Publishers) p 17 cond-mat/0010376

[4] Rice T M 2001 High Magnetic Fields Lecture notes in physics 595 eds. Berthier C, Lévy L P and Martinez G (Berlin: Springer) p 139 http://www.springerlink. com/link. asp?id=vklm1hrd82j665wv

[5] Honecker A 2003 habilitation thesis http://www.tu-bs.de/ honecker/papers/habil.ps.gz

[6] Oshikawa M, Yamanaka M and Affleck I 1997 Phys. Rev. Lett. 781984

[7] Honecker A 1999 Phys. Rev. B 596790

[8] Majumdar C K and Ghosh D K 1969 J. Math. Phys. 101388

[9] Majumdar C K and Ghosh D K 1969 J. Math. Phys. 101399

[10] Majumdar C K 1970 J. Phys. C: Solid State Phys. 3911

[11] Tonegawa T and Harada I 1987 J. Phys. Soc. Japan 562153

[12] Cabra D C, Honecker A and Pujol P 2000 Eur. Phys. J. B 1355

[13] Okunishi K and Tonegawa T 2003 J. Phys. Soc. Japan 72479

[14] White S R and Affleck I 1996 Phys. Rev. B 549862

[15] Ivanov N B and Richter J 1997 Phys. Lett. A 232308

[16] Richter J, Ivanov N B and Schulenburg J 1998 J. Phys.: Condens. Matter 103635

[17] Shastry B S and Sutherland B 1981 Physica 1081069

[18] Koga A, Okunishi K and Kawakami N 2000 Phys. Rev. B 625558

[19] Schulenburg J and Richter J 2002 Phys. Rev. 65054420 
[20] Yang M S and Mütter K-H 1997 Z. Phys. B 104117

[21] Honecker A 1999 J. Phys.: Condens. Matter 114697

[22] Zhitomirsky M E and Nikuni T 1998 Phys. Rev. B 575013

[23] Sandvik A W 1999 Phys. Rev. B 59 R14157

[24] Nishimori H and Miyashita S 1986 J. Phys. Soc. Japan 554448

[25] Chubukov A V and Golosov D I 1991 J. Phys.: Condens. Matter 369

[26] Bernu B, Lecheminant P, Lhuillier C and Pierre L 1994 Phys. Rev. B 5010048

[27] Miyashita S 1986 J. Phys. Soc. Japan 553605

[28] Hida K 2001 J. Phys. Soc. Japan 703673

[29] Schulenburg J, Honecker A, Schnack J, Richter J and Schmidt H-J 2002 Phys. Rev. Lett. 88167207

[30] Waldtmann Ch and Everts H-U 1999 private communication

[31] Waldtmann Ch, Everts H-U, Bernu B, Lhuillier C, Sindzingre P, Lecheminant P and Pierre L 1998 Eur. Phys. J. B 2501

[32] Mila F 1998 Phys. Rev. Lett. 812356

[33] Cabra D C, Grynberg M D, Holdsworth P C W and Pujol P 2002 Phys. Rev. B 65094418

[34] Zhitomirsky M E 2002 Phys. Rev. Lett. 88057204

[35] Shender E F and Holdsworth P C W 1996 Fluctuations and Order ed. Millonas M (New York: Springer-Verlag) p 259

[36] Cabra D C, Grynberg M D, Holdsworth P C W, Honecker A, Pujol P, Richter J and Schulenburg $\mathrm{J} 2003$ in preparation

[37] Moessner R and Sondhi S L 2001 Phys. Rev. B 63224401

[38] Moessner R, Sondhi S L and Chandra P 2001 Phys. Rev. B 64144416

[39] Richter J, Schulenburg J, Honecker A, Schnack J and Schmidt H-J 2003 submitted to J. Phys.: Condens. Matter

[40] Momoi T and Totsuka K 2000 Phys. Rev. B 613231

[41] Oshikawa M 2000 Phys. Rev. Lett. 841535

[42] Kageyama H, Yoshimura K, Stern R, Mushnikov N V, Onizuka K, Kato M, Kosuge K, Slichter C P, Goto T and Ueda Y 1999 Phys. Rev. Lett. 823168

[43] Onizuka K, Kageyama H, Narumi Y, Kindo K, Ueda Y and Goto T 2000 J. Phys. Soc. Japan 69 1016

[44] Miyahara S and Ueda K J. Phys.: Condens. Matter 200315 R327 